\title{
Original und Digitalisat gemeinsam denken!
}

\section{Katastrophen rütteln wach}

In den vergangenen 20 Jahren hat das Thema Schutz und Erhaltung der Originale unserer schriftlichen Überlieferung in der Öffentlichkeit und in der Politik eine sichtbare Wahrnehmung und Positionierung erfahren. Ein langfristiger Prozess zur Umsetzung abgestimmter Handlungsempfehlungen auf Bundes-, Länder- und Einrichtungsebene wurde eingeleitet und zeigt inzwischen erste zählbare Erfolge. Noch an der Schwelle zum 21. Jahrhundert war dieser Prozess in dieser Ausprägung nicht vorstellbar gewesen.

$\mathrm{Zu}$ der Entwicklung beigetragen haben die unvergesslichen Bilder von Katastrophen, die nach dem Jahr 2000 in Deutschland leider gehäuft auftraten und zu erheblichen Bestandsschäden und Bestandsverlusten in mehreren Kultureinrichtungen geführt haben. Dazu gehören der Brand der Herzogin Anna Amalia Bibliothek in Weimar am 2. September 2004, der Einsturz des Kölner Stadtarchivs am 3. März 2009 oder die Hochwasserschäden in verschiedenen Bibliotheken und Archiven im August 2002 (Elbe und ihre Nebenflüsse), im Mai 2010 (Oder-Neiße-Gebiet) oder Anfang Juni 2013 (Mitteleuropa). Die durch diese Katastrophen verursachten konservatorischen Erfordernisse sind zum Teil noch heute präsent.

Weiterhin bedurfte es des gemeinsamen und starken Engagements von Vertretern aus dem Bibliotheks- und Archivwesen, um ausgehend von diesen Katastrophen der Politik den drängenden Handlungsbedarf aufzuzeigen und grundsätzliche und langfristige Lösungsansätze für den Schutz und Erhalt der Originale unserer schriftlichen Überlieferung einzufordern.

\section{Sensibilisierung der Politik für den Originalerhalt}

Auf Fachebene war an diesem Prozess maßgeblich die im Jahr 2001 gegründete „Allianz Schriftliches Kulturgut Erhalten“ beteiligt. In ihrer im Jahr 2008 verabschiedeten und im Jahr 2009 publizierten Denkschrift Zukunft Bewahren gab sie sieben Handlungsempfehlungen an Bund und Länder. ${ }^{1}$ Drei Kernempfehlungen

1 Zukunft bewahren. Eine Denkschrift der Allianz zur Erhaltung des schriftlichen Kulturguts. Hrsg. von Barbara Schneider-Kempf. Text: Thomas Bürger, Bernd Kappelhoff, Michael Knoche

ə Open Access. (C) 2018 Ursula Hartwieg, Michael Vogel, publiziert von De Gruyter. (c) BY-NC-ND Dieses Werk ist lizenziert unter der Creative Commons Attribution-NonCommercial-NoDerivatives 4.0 Lizenz. 
waren eine zwischen Bund und Ländern abgestimmte nationale Konzeption zur Erhaltung des schriftlichen Kulturguts, die Stärkung des Originalerhalts von national bedeutsamem Kulturgut durch den Bund mit jährlich zehn Millionen Euro und die Einrichtung einer zentralen Koordinierungsstelle bei einer vorhandenen Einrichtung. Damit wurden wichtige Punkte des Abschlussberichtes der Enquete-Kommission „Kultur in Deutschland“ vom 11. Dezember 2007² aufgegriffen und noch einmal verstärkt. Mit der feierlichen Übergabe dieser Denkschrift an den damaligen Bundespräsidenten Horst Köhler am 28. April 2009 erreichte die „Allianz“ ein nachhaltiges Echo in der Politik, wie sich an der nachfolgenden Auswahl von Ereignissen in chronologischer Aufstellung ablesen lässt.

Tab. 1: Ereignistabelle (Referenzen am Ende des Beitrags).

\begin{tabular}{|c|c|}
\hline Datum & Ereignis \\
\hline 26.10 .2009 & $\begin{array}{l}\text { Koalitionsvertrag von CDU, CSU und FDP auf Bundesebene: „Gemeinsam mit } \\
\text { den Ländern wollen wir ein nationales Bestandserhaltungskonzept für gefähr- } \\
\text { detes schriftliches Kulturgut erarbeiten. Zum verstärkten Schutz schriftlichen } \\
\text { Kulturgutes wird eine Koordinierungsstelle eingerichtet.“ [1] }\end{array}$ \\
\hline 5.11 .2010 & $\begin{array}{l}\text { Pressemitteilung „Bund und Länder fördern Erhalt schriftlichen Kulturguts“ [2] } \\
\text { erstmals mit } 600000 \text { Euro (... und setzen die damit verbundene Modellpro- } \\
\text { jektförderung in den Folgejahren kontinuierlich fort.) }\end{array}$ \\
\hline 22.7 .2011 & $\begin{array}{l}\text { Gründung der Koordinierungsstelle für die Erhaltung des schriftlichen Kultur- } \\
\text { guts (KEK) [3] durch Bund und Länder auf vorerst fünf Jahre mit einer jährli- } \\
\text { chen Finanzierung in Höhe von } 600000 \text { Euro: } 500000 \text { Euro aus dem Haushalt } \\
\text { des Bundes, } 100000 \text { Euro für die Länder aus dem Etat der Kulturstiftung der } \\
\text { Länder (KSL) }\end{array}$ \\
\hline 27.11 .2013 & $\begin{array}{l}\text { Koalitionsvertrag von CDU, CSU und SPD auf Bundesebene: „Die Erhaltung } \\
\text { des schriftlichen Kulturgutes ist eine gesamtstaatliche Aufgabe. Die entspre- } \\
\text { chende Koordinierungsstelle bei der Staatsbibliothek zu Berlin wird auf Basis } \\
\text { einer bereits geplanten Evaluierung und in Abstimmung mit den Ländern, ge- } \\
\text { gebenenfalls über ein Bund-Länder-Förderprogramm, über } 2015 \text { hinaus fort- } \\
\text { geführt.“ [4] }\end{array}$ \\
\hline Herbst 2014 & $\begin{array}{l}\text { Positive Evaluierung der KEK in archiv- und bibliotheksfachlicher sowie in or- } \\
\text { ganisatorischer Hinsicht }\end{array}$ \\
\hline
\end{tabular}

u. Barbara Schneider-Kempf. Berlin: Staatsbibliothek zu Berlin - Preußischer Kulturbesitz 2009. http://www.allianz-kulturgut.de/fileadmin/user_upload/Allianz_Kulturgut/dokumente/ 2009_Allianz_Denkschrift_gedruckt.pdf. Alle aufgeführten Internetressourcen wurden zuletzt am 27. Dezember 2017 aufgerufen.

2 Schlussbericht der Enquete-Kommission „Kultur in Deutschland“, 11. Dezember 2007 [am 13. Dezember 2007 im Plenum des Deutschen Bundestages debattiert]. https://dip21.bundestag. de/dip21/btd/16/070/1607000.pdf. 


\begin{tabular}{ll}
\hline Datum & Ereignis \\
\hline 9.10.2015 & $\begin{array}{l}\text { Pressemitteilung: KEK legt Bund, Ländern und Kommunen auftragsgemäß die } \\
\text { Bundesweiten Handlungsempfehlungen zur Erhaltung des schriftlichen Kul- } \\
\text { turguts in Deutschland vor und schließt mit dieser nationalen Bestandserhal- } \\
\text { tungskonzeption die Pilotphase ab [5] }\end{array}$ \\
\hline 11.11.2016 & $\begin{array}{l}\text { Pressemitteilung: Kulturstaatsministerin Monika Grütters unterstützt die Er- } \\
\text { haltung des schriftlichen Kulturguts 2017 mit bis zu einer Million Euro [6] }\end{array}$ \\
\hline 28.7.2017 & Pressemitteilung: BKM-Sonderprogramm fördert auf der Basis von Empfeh- \\
& $\begin{array}{l}\text { lungen des Fachbeirats der KEK bundesweit Projekte in Archiven und Biblio- } \\
\text { theken [7] }\end{array}$ \\
\hline 11.8.2017 & $\begin{array}{l}\text { Wiederauflage des BKM-Sonderprogramms für die Erhaltung des schriftlichen } \\
\text { Kulturguts mit einer Million Euro für 2018 geplant [8] }\end{array}$ \\
\hline 14.8.2017 & $\begin{array}{l}\text { Pressemitteilung: Förderung von 79 Projekten bundesweit in Höhe von 1,5 } \\
\text { Millionen Euro für Überlieferungssicherung durch zwei Förderlinien, einer- } \\
\text { seits die inzwischen klassische Modellprojektförderung (Themenschwerpunkt } \\
\text { „Das besondere Format“ mit 34 Vorhaben zur exemplarischen Sicherung von } \\
\text { Sonderformaten), andererseits das BKM-Sonderprogramm (45 Projekte im Be- } \\
\text { reich Mengenverfahren Entsäuerung, Schutzverpackung und Trockenreini- } \\
\text { gung) [9] }\end{array}$ \\
\hline
\end{tabular}

\section{„Wegweiser“ in das nächste Jahrhundert}

Mit den Bundesweiten Handlungsempfehlungen wurde der Politik ein schlüssiges Konzept übergeben, das sehr langfristig über einen Zeitraum von 100 Jahren ausgelegt ${ }^{3}$ und trotzdem überschaubar und berechenbar ist, exakte Aussagen zum Mittelbedarf und -einsatz ermöglicht und deshalb variabel in kurz-, mittelund langfristige Finanzplanungen auf Bundes-, Länder- und Einrichtungsebene integrierbar ist. Es ist außerdem flexibel hinsichtlich der Bildung von kurzfristig durchführbaren und finanzierbaren Schwerpunkten, jederzeit transparent darstellbar sowie über Kennziffern abrechenbar.

3 Koordinierungsstelle für die Erhaltung des schriftlichen Kulturguts: Die Erhaltung des schriftlichen Kulturguts in Archiven und Bibliotheken in Deutschland. Bundesweite Handlungsempfehlungen für die Beauftragte der Bundesregierung für Kultur und Medien (BKM) und die Kultusministerkonferenz (KMK). Berlin: Koordinierungsstelle für die Erhaltung des schriftlichen Kulturguts an der Staatsbibliothek zu Berlin - Preußischer Kulturbesitz 2015. http://kek-spk.de/fileadmin/user_upload/pdf_Downloads/KEK_Bundesweite_Handlungsempfehlungen.pdf. In den Bundesweiten Handlungsempfehlungen wird eine Bedarfssumme in Höhe von 6,3 Milliarden Euro bezogen auf den Zeitraum von 100 Jahren genannt, was einer jährlichen Summe von 63 Millionen Euro für den Originalerhalt entspricht. Dieser jährliche Bedarf teilt sich wie folgt auf: Sparte Archive - zwei Drittel (42 Millionen Euro) und Sparte Bibliotheken - ein Drittel (21 Millionen Euro). 
Vergleichbar langfristige Planungen über den Zeitraum von 100 Jahren gibt es im Bibliothekswesen bisher nicht. Eine solche Sichtweise ist Neuland und verlangt die Bereitschaft von Politik, Förderern und Geldgebern, von den Kultureinrichtungen und ihren Trägern sowie von den Dienstleistern, konsequent langfristig zu denken und sich auf eine planvolle und zielführende Lösung in kleinen Schritten einzulassen.

Dabei ist festzuhalten, dass die Erfordernisse des Originalerhalts bei Weitem nicht einem so hohen Innovations- oder Erneuerungsdruck folgen, wie er vielleicht in anderen Bereichen, beispielsweise der Digitalen Bibliothek bestehen mag. Diese Beständigkeit im Kontext mit klaren restaurierungsethischen Grundsätzen und definierten handwerklichen und technologischen Prämissen ist aber gerade der langfristige Planungsvorteil für den Originalerhalt.

Allerdings spielt der Faktor Zeit eine keinesfalls $\mathrm{zu}$ vernachlässigende Rolle, da sich endogene Wirkmechanismen (wie Tinten-, Farb-, Säurefraß) und ungünstige exogene Bedingungen (wie ungenügendes Raumklima, unsachgemäße Lagerung, häufige Benutzung fragiler Objekte) mit der Zeit verschärfen, sogar zur Zerstörung der betroffenen Originale und insbesondere zur Verteuerung der notwendigen konservatorischen oder restauratorischen Maßnahmen führen können.

Insofern wäre es keine Lösung, die nationale Aufgabe Originalerhalt in die Zukunft zu verschieben und künftigen Generationen zuzuordnen. Vielmehr ist kontinuierliches und zielstrebiges Engagement erforderlich. Dabei ist von Vorteil, dass eine umfangreiche Palette erprobter konservatorischer und restauratorischer Maßnahmen zur Verfügung steht. Ohne Frage wird es auf diesem Gebiet auch Weiterentwicklungen und neue Forschungen geben. Mit Spannung erwartet werden beispielsweise kostengünstige Mengenverfahren zur Festigung von sauren, sehr fragilen oder bereits brüchigen Papieren.

Bestandserhaltung ist per se verpflichtet, beharrlich für den Originalerhalt einzutreten, die damit verbundenen Maßnahmen hoch zu priorisieren und konsequent für die Bindung der notwendigen Ressourcen einzutreten. Das darf aber nicht damit verwechselt werden, dass Bestandserhaltung etwa mit einem veralteten Verständnis von Bibliothek als bloßer ,Bücheransammlung‘ einhergeht. Vielmehr geht es darum, die Originale des schriftlichen Kulturguts für kommende Generationen zu bewahren und zu überliefern, heute und in Zukunft als Vorlage für die Digitalisierung bereitzuhalten und Sicherheit für die Langzeitverfügbarkeit zu schaffen, allein oder im Kontext mit digitalen Präsentationen als materielle Erlebnis- und insbesondere Forschungsobjekte anzubieten, in Ausstellungen dreidimensional mit der den Originalen anhaftenden Aura und Patina darzustellen, für neue Forschungsmethoden und die Beantwortung neuer Fragestellungen dauerhaft verfügbar zu halten. 


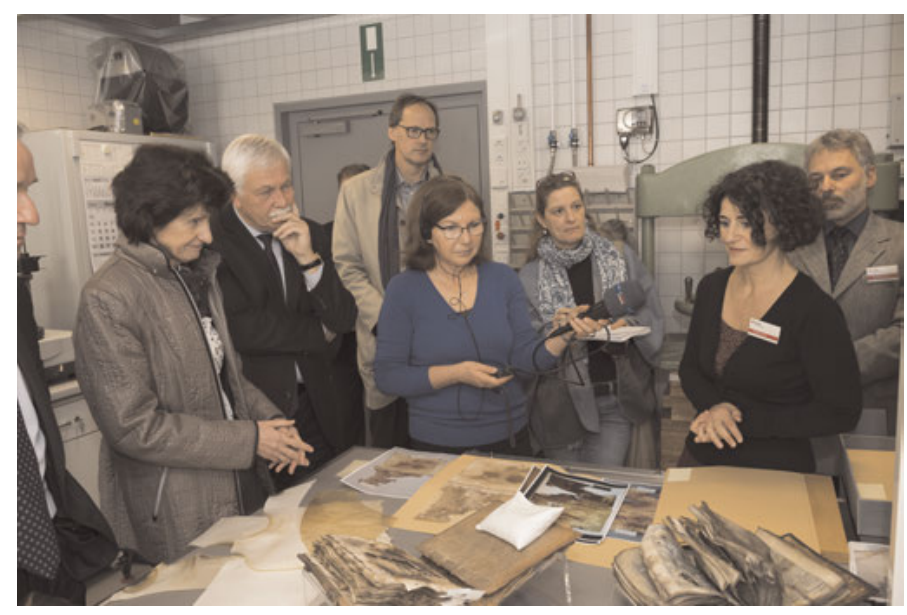

Abb. 1: Am 29. Oktober 2015 nehmen Dr. Eva-Maria Stange, Staatsministerin für Wissenschaft und Kunst (links im Bild), und Prof. Thomas Bürger in der SLUB die neue Klimakammer zur Pergamentrestaurierung in Betrieb und informieren sich über komplexe Schadensbilder an wertvollen Handschriften und über Restaurierungsmöglichkeiten, Foto: (C SLUB, Ahlers.

\section{Chancen im Wandel}

Nach Lankes werden sich Bibliotheken sehr schnell neuen Herausforderungen stellen müssen. ${ }^{4}$ Der eingeleitete Trend für wissenschaftliche Bibliotheken hin zu Kommunikationszentren und Lernräumen verbildlicht Orte, an denen Menschen arbeiten, kommunizieren, neue Ideen entwickeln oder sich einfach nur in einer für sie angenehmen Atmosphäre aufhalten wollen. ${ }^{5}$ In der Zukunft geht es nach Lankes auch verstärkt darum, Communitys für Bibliotheken zu etablieren, darin möglichst viele aktive Mitglieder zu binden und die Kommunikation mit und zwischen ihnen in den Fokus zu rücken. ${ }^{6}$

4 Vgl. Lankes, Richard David: Erwarten Sie mehr! Verlangen Sie bessere Bibliotheken für eine komplexer gewordene Welt. Berlin: Simon Verlag für Bibliothekswissen 2017.

5 Vgl. Balleer, Jessica: Wo Bibliotheken aussehen wie Luxushotels und Oasen der Ruhe sind ein Besuch in Belgien und den Niederlanden. In: RP online vom 22. September 2016.

http://www.rp-online.de/kultur/die-schoensten-bibliotheken-der-zukunft-aid-1.6276797; Stampfl, Nora: Die Bibliothek als Demokratiemotor. In: The European vom 21. September 2016. http://www.theeuropean.de/nora-stampfl/11295-zukunft-der-bibliotheken.

6 Vgl. Lankes, Erwarten Sie mehr (wie Anm. 4). 
Es wird aber wohl keine uniforme Community geben können, vielmehr werden unterschiedliche Mitgliederzahlen ganz unterschiedlichen Interessengruppen angehören und verschiedene Ziele verfolgen. Eines dieser Ziele wird mit Sicherheit die tief gehende Befassung mit den Originalen unserer kulturellen Überlieferung und ihrer Materialität sein. Dass dies sehr realistisch ist, hat beispielsweise die „Henry Arnhold Dresden Summer School“ 2017 gezeigt. In unserer zunehmend digitalen Welt und ihren Netzwerken fest verwurzelte junge Akademiker haben die Gelegenheit genutzt, sich unter anderem in der Restaurierungswerkstatt der SLUB Dresden intensiv über die Restaurierung zum Ende des Zweiten Weltkriegs schwerstgeschädigter Pergamentobjekte zu informieren und dabei nicht nur zu sehen, zu beobachten und zuzuhören, sondern auch selbst zu tasten und sogar zu riechen. Alle haben ihre so mit verschiedenen Sinnesorganen gewonnenen Eindrücke und die Möglichkeit, derart die Aura der Objekte $\mathrm{zu}$ verinnerlichen, als deutlichen Informationsgewinn eingestuft und diese Veranstaltung mit Bestnoten bewertet.

Materialität anhand ihrer Originale zu vermitteln, ist für Bibliotheken eine große Chance und öffnet für Bibliotheken selbst die Möglichkeit, nicht nur Mitglied, sondern Triebkraft in der Forschungscommunity zu sein. Allerdings müssen die dafür erforderlichen gerätetechnischen Voraussetzungen bzw. die Möglichkeiten zur Interaktion geschaffen oder ausgebaut werden, was am besten über gut funktionierende Netzwerke mit Hochschulen, Forschungseinrichtungen und vor allem interessierten Nutzern erfolgt.

\section{Zweigleisig in die Zukunft: original und digital}

Natürlich liegt es auf der Hand, dieses Potenzial mit einer weiteren Stärke und strategischen Zukunftsaufgabe der Bibliotheken eng zu verknüpfen, nämlich Original und Digitalisat gemeinsam zu denken. Das ist nicht gleichzusetzen mit einer bloß dienenden Funktion des Originals für die (eventuell wiederholte) Erzeugung von Digitalisaten, die es natürlich auch gibt und die künftig aus Kostengründen im Kontext mit der elektronischen Langzeitarchivierung sicher noch viel stärker beachtet werden muss, sondern setzt die Akzeptanz beider Formen voraus und die Bereitschaft, als Zukunftsaufgabe beide Formen zu überliefern und mögliche Synergieeffekte sinnvoll zu nutzen. ${ }^{7}$ So können durch

7 Vgl. Knoche, Michael: In guter Ordnung, aber schlechter Verfassung. In: Frankfurter Allgemeine Zeitung vom 18. Juli 2017. http://www.faz.net/aktuell/feuilleton/debatten/buecherschaetze-muessen-digitalisiert-und-restauriert-werden-15110277.html. 
Digitalisate einerseits Originale geschont und andererseits schriftliches Kulturgut der breiten Öffentlichkeit nahegebracht werden.

Für Handschriften oder vor ca. 1850 erschienene Druckwerke ist das Miteinander von Original und Digitalisat und somit die Erhaltung der Originale breiter Konsens. Für danach erschienene Druckwerke haben die „Allianz Schriftliches Kulturgut Erhalten“ in ihrer Denkschrift Zukunft Bewahren und die Koordinierungsstelle für die Erhaltung des schriftlichen Kulturguts (KEK) in den Bundesweiten Handlungsempfehlungen eine gemeinsame Sprachregelung zum Originalerhalt verwendet: Mindestens ein Exemplar eines jeden in Deutschland erschienenen oder sich auf Deutschland beziehenden Titels soll langfristig erhalten werden. Wenn es länderübergreifend gelingt, diesen Anspruch nachhaltig in der Praxis umzusetzen, wäre bereits viel erreicht. Dabei fasziniert die Vorstellung, für alle konservatorisch vollständig bearbeiteten und optimal gelagerten Exemplare konsequent ein Digitalisat zur Benutzung anzubieten und beispielsweise in die Deutsche Digitale Bibliothek ${ }^{8}$ oder in die Europeana ${ }^{9}$ einzubinden. Diese Vorstellung in die Tat umsetzen zu wollen, bedeutet auch aus Sicht der Bestandserhaltung, eine umfassende Förderung der Digitalen Bibliothek zu unterstützen, um für diesen Zeitraum ebenso die systematische Digitalisierung der Bestände zu ermöglichen und damit die abgestimmte Bearbeitung von Bestandsgruppen. Hier sind gemeinsame strategische Ziele zu formulieren und die Politik ist gefordert, übergreifende Förderlinien für Originalerhalt und Digitalisierung zu initiieren, denn allein auf Einrichtungs- und Landesebene kann diese gewaltige Zukunftsaufgabe nicht bewältigt werden und sie verlangt nach einer breiten Koordinierung.

\section{Herausforderung saures Papier}

Ein Exemplar eines jeden Titels aus dem Zeitraum nach 1850 zuverlässig zu überliefern, stellt mathematisch die Minimalvariante dar. Bei genauer Betrachtung gelangt man sehr schnell zu dem Schluss, dass dies aus verschiedenen Gründen nicht ausreichen kann und der Zusatz „mindestens ein Exemplar“ zwingend erfolgen muss und eine Obergrenze nicht begründbar ist. Zunächst stellt sich die Frage, was im Original überliefert werden soll: Steht das Gedruckte im Buchblock im Vordergrund, spielen Buchhandelseinbände eine Rolle oder sind Bibliothekseinbände genauso akzeptabel, weil vielleicht stabi-

$8 \mathrm{https} / /$ www.deutsche-digitale-bibliothek.de/.

9 https://www.europeana.eu/portal/de. 
ler, sind Autografen und handschriftliche Einträge insgesamt bedeutungsvoll oder sind Provenienzen und besondere Sammlungszusammenhänge zu beachten und wie kann und soll der sehr heterogene Erhaltungszustand vergleichend bewertet werden? Wer soll es leisten, per Autopsie das ,am besten erhaltene' oder ,am besten geeignete، Exemplar in seiner Gesamtheit einzuschätzen und welche Informationen werden in der Zukunft relevant sein? Welche dieser zusätzlichen oder besonderen, auf jeden Fall exemplarspezifischen Informationen werden in die Digitale Bibliothek überführt und sind dauerhaft online abrufbar?

Dass sich Buchexemplare einer identischen Auflage auf ganz vielfältige Weise voneinander unterscheiden können, ist hinlänglich bekannt. Das zeigen auch erste Vergleiche innerhalb der so genannten „Testothek“, die im Rahmen eines KEK-Modellprojekts in Kooperation zwischen SLUB Dresden, Deutscher Nationalbibliothek, Staatsbibliothek zu Berlin und Bayerischer Staatsbibliothek aufgebaut und zunächst für 180 Titel aus dem Erscheinungszeitraum 1850 bis 1990 je sechs Exemplare einer definierten Auflage umfassen wird.

Auch wissen wir derzeitig noch vergleichsweise wenig über die nachhaltige Wirkung der verschiedenen am Markt etablierten Massenentsäuerungsverfahren oder ihre möglichen langfristigen Nebenwirkungen. Um dazu in allen Punkten gesicherte Aussagen treffen zu können, werden in Zukunft unbehandelte, also nicht entsäuerte Vergleichsexemplare in einem Umfang notwendig sein, der weit über den der „Testothek“ hinausgeht. Mit Sicherheit müssen in der Zukunft möglicherweise neue oder technologisch weiterentwickelte oder chemisch veränderte Verfahren zur Massenentsäuerung oder zur Papierfestigung mitberücksichtigt werden.

Genau zu überlegen und abzuwägen wird sein, welche Benutzungsforderungen künftig an die Originale gestellt werden. Mittelfristig werden das auch weiterhin noch die klassischen Anforderungen Ortsleihe, Fernleihe, Lieferdienste, Ausstellungswesen oder Leihverkehr für Ausstellungen und die eventuell wiederholte Digitalisierung zur Onlinestellung sein. Dafür sollten aus konservatorischen Gründen die langfristig zu erhaltenden, massenentsäuerten Exemplare mit Ausnahme zur Digitalisierung nicht herangezogen werden, da sonst weitere Schäden hervorgerufen oder Verluste eintreten können. Ohnehin sind für eine vollständige und gesicherte Überlieferung eine in die Zukunft gerichtete Risikoabschätzung und ein abgestimmtes Nebeneinander von physischen Exemplaren und Digitalisaten unabdingbar. 


\section{Risiken bedenken - Sicherheit wählen}

Die Palette möglicher Gefährdungen sowohl für die Originale als auch die digital vorliegenden Informationen wird zunehmend breiter. Neben der Hochwasser- oder Brandgefahr werden uns die Auswirkungen des Klimawandels mit Extremereignissen wie Hitzeperioden und den ungünstigen Folgen für das Raumklima, mit immer häufiger auftretenden Unwettern bis hin zu Tornados oder einem sich ändernden Schädlingsspektrum vor neue Herausforderungen stellen. Die stabile Sicherstellung der Energieversorgung, das Management bei einem möglichen Blackout oder die Abwehr von denkbaren Cyberattacken müssen vorausgedacht werden. Terrorismus und Krieg wären die schrecklichsten Szenarien und am wenigsten kalkulierbar.

Nur das Miteinander von den Originalen unserer schriftlichen Überlieferung und ihren Digitalisaten lassen zumindest die aus Naturgewalten und technischen Störfällen resultierenden möglichen Risiken dahingehend beherrschbar erscheinen, dass ein Totalverlust von Informationen und Wissen vermeidbar bleibt. Die eigentliche Herausforderung wird allerdings darin bestehen, das nicht nur für einige Jahre oder Jahrzehnte, sondern für Jahrhunderte zuverlässig sicherzustellen! Auch aus diesem Aspekt heraus sind Originalerhalt und Digitalisierung als sich wechselseitig ergänzende und gleichzeitig kooperierende strategische Felder zu betrachten, die beide einer grundständigen Finanzierung bedürfen.

Schließlich muss die Frage, ob kulturelle Vielfalt in unserer Gesellschaft und die Integration von Menschen aus anderen Kulturkreisen in unsere Gesellschaft neben gesicherten digitalen Quellen auch Originale braucht, mit Ja beantwortet werden. Nur so kann garantiert werden, dass in der Zukunft alle Bürger, Politiker und Forscher in der Lage sein werden, das Leben und Streiten des Heute nachzuvollziehen und zu verstehen, woher wir kommen und welche unsere Wurzeln sind. 


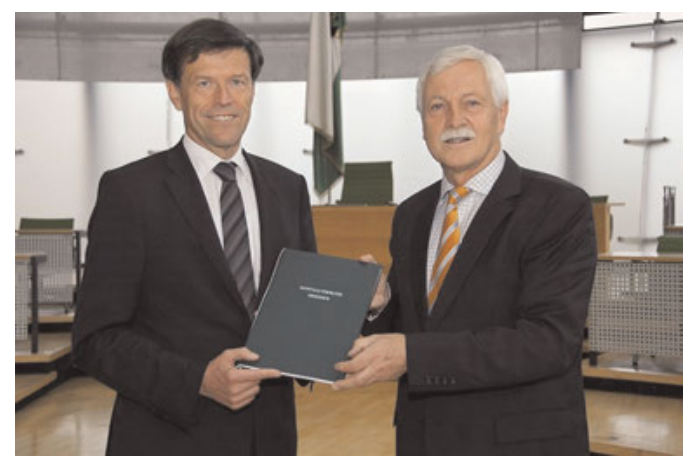

Abb. 2: Gemeinsam präsentieren Dr. Matthias Rößler, Präsident des Sächsischen Landtags, und Prof. Thomas Bürger am 23. September $2011 \mathrm{im}$ Sächsischen Landtag die soeben von elf Dresdner Kultureinrichtungen unterzeichneten Gründungsdokumente des Notfallverbunds Dresden, Foto: @ SLUB, Rous.

\section{Einrichtungen}

Allianz Schriftliches Kulturgut Erhalten: http://www.allianz-kulturgut.de/die-allianz/ Henry Arnhold Dresden Summer School 2017: http://dresdensummerschool.de/ Koordinierungsstelle für die Erhaltung des schriftlichen Kulturguts (KEK):

http://www.kek-spk.de

\section{Referenzen zur Ereignistabelle}

[1] Wachstum. Bildung. Zusammenhalt. Koalitionsvertrag von CDU, CSU und FDP. 17. Legislaturperiode, 26. Oktober 2009, S. 96. http://www.csu.de/common/_migrated/ csucontent/091026_koalitionsvertrag.pdf.

[2] Kulturstaatsminister Bernd Neumann: Bund und Länder fördern Erhalt schriftlichen Kulturguts. Pressemitteilung 417 des Presse- und Informationsamtes der Bundesregierung, 5. November 2010. https://www.bundesregierung.de/ContentArchiv/DE/ Archiv17/Pressemitteilungen/BPA/2010/11/2010-11-05-bkm-bund-laender.html.

[3] Vgl. Koordinierungsstelle für die Erhaltung des schriftlichen Kulturguts nimmt ihre Arbeit auf: Ein wichtiger Schritt für das kulturelle Erbe. Pressemitteilung 267 des Presse- und Informationsamtes der Bundesregierung, 22. Juli 2011. https://www.bundesregierung.de/ ContentArchiv/DE/Archiv17/Pressemitteilungen/BPA/2011/07/2011-07-22-bkm-kulturguterbe.html.

[4] Deutschlands Zukunft gestalten. Koalitionsvertrag zwischen CDU, CSU und SPD. 18. Legislaturperiode, 27. November 2013, S. 131. https://tinyurl.com/ya4oetmw.

[5] Vgl. Gemeinsame Pressemitteilung von BKM und KMK. Bund, Länder und Kommunen unterstreichen gemeinsame Verantwortung für den Kulturgutschutz in Deutschland. 
Pressemitteilung 353 des Presse- und Informationsamtes der Bundesregierung, 9. Oktober 2015. https://www.bundesregierung.de/Content/DE/Pressemitteilungen/ BPA/2015/10/2015-10-09-verantwortung-kulturgutschutz.html.

[6] Vgl. 280 Millionen Euro zusätzlich für den Bundeskulturhaushalt - Grütters: Schöne Bestätigung für den Wert der Kultur. Pressemitteilung 407 des Presse- und Informationsamtes der Bundesregierung, 11. November 2016. https://www.bundesregierung.de/ Content/DE/Pressemitteilungen/BPA/2016/11/2016-11-11-bkm-haushalt.html.

[7] Vgl. Grütters: Sonderprogramm fördert langfristige Erhaltung unseres schriftlichen Kulturerbes. Pressemitteilung 279 des Presse- und Informationsamtes der Bundesregierung, 28. Juli 2017. https://www.bundesregierung.de/Content/DE/Pressemitteilungen/BPA/ 2017/07/2017-07-28-bkm-schriftliches-kulturerbe.html.

[8] Vgl. Entwurf zum Bundeshaushaltsplan 2018. Einzelplan 04. Bundeskanzlerin und Bundeskanzleramt, Anlage zur Drucksache 18/13000, S. 63. http://dipbt.bundestag.de/ doc/btd/18/130/1813000.pdf.

[9] Vgl. Originalerhalt im Sonderformat. 1,5 Millionen Euro Fördermittel für Archive und Bibliotheken. Pressemitteilung der KEK, 14. August 2017. https://tinyurl.com/yd27r5sl. 